\title{
An Interesting Case of Intestinal Obstruction
}

\author{
Gp Capt KLS Kumar ${ }^{*}$ Air Cmde DP Joshi ${ }^{+}$, AVM (Retd) JK De, vsm ${ }^{\#}$
}

MJAFI 2007; 63 : 73-74

Key Words: Intestinal obstruction; Internal herniation; Jejunal mesentery

\section{Introduction}

Thte nternal hernias due to postoperative adhesions and bands are uncommon causes of small bowel obstruction. In addition internal hernias due to congenital abnormalities or surgical defects in the mesentery causing bowel obstruction are very rare [1].

An interesting case of intestinal obstruction as a result of internal herniation through a congenital defect in the jejunal mesentery is reported.

\section{Case Report}

A 28 year old male patient presented to a peripheral hospital with the history of pain abdomen of two weeks duration and vomiting of two days duration. Clinical examination was unremarkable except for signs of mild dehydration. Plain radiograph of abdomen showed dilatation of duodenum and the proximal jejunal loops. Ultrasound examination of abdomen revealed, an aperistaltic segment of a loop of jejunum immediately following the dilated loop of jejunum. A provisional diagnosis of sub acute intestinal obstruction was made and the patient was treated conservatively with nasogastric tube aspiration and intravenous fluids. He recovered and was discharged from the hospital within 96 hours of admission. However after 72 hours, the patient reported back to the hospital with the recurrence of symptoms. Again the patient was treated with nasogastric tube aspiration and intravenous fluids. He made good recovery. However, in view of recurrence of symptoms within 72 hours of initial episode, he was sent to referral hospital for further management where the patient described the onset of pain as insidious, centrally located without any history of radiation. It was dull aching associated with bloating sensation and intermittent colicky attacks of pain. The vomitus contained bile. The patient also gave past history of scleroderma of four years duration. On examination, the patient was looking ill with signs of mild dehydration. The skin showed the features of scleroderma. Vital signs were stable. Local examination of abdomen revealed minimal distension of upper half of abdomen. There was no visible peristalsis, localized area of tenderness, guarding, rigidity, or evidence of free fluid in the peritoneal cavity. Intestinal sounds were normal. Digital rectal examination was normal. Routine haematological and biochemical investigations were within normal limits. Plain radiograph of abdomen showed the dilatation of duodenum and proximal jejunal loops (Fig. 1). In view of the sub acute nature of the episode, the patient was kept under close observation. He continued to have persistent large volumes of naso gastric tube aspiration. The mantoux test was non reactive.The computed tomography (CT) scan of abdomen was suggestive of upper small bowel obstruction.

During hospitalisation, the patient developed recurrence of symptoms and was taken up for exploratory laparotomy. At laparotomy, stomach, duodenum and proximal $20 \mathrm{~cm}$ of

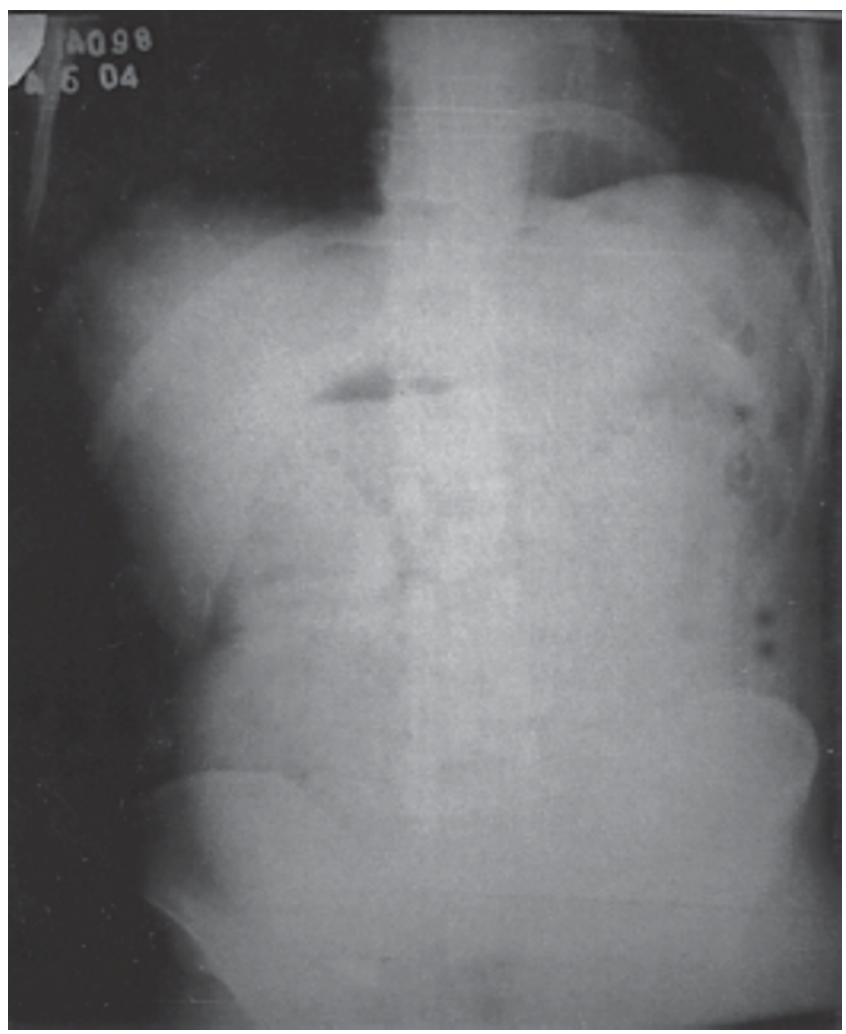

Fig. 1: Plain radiograph of abdomen showing dilatation of duodenum and proximal jejunal loops

"Senior Advisor (Surgery), Command Hospital (Air Force), Bangalore. ${ }^{+}$Consultant (Surgery \& Urology) Command Hospital (Central Command) Lucknow. "Ex Commandant, Command Hospital (Air Force), Bangalore. 


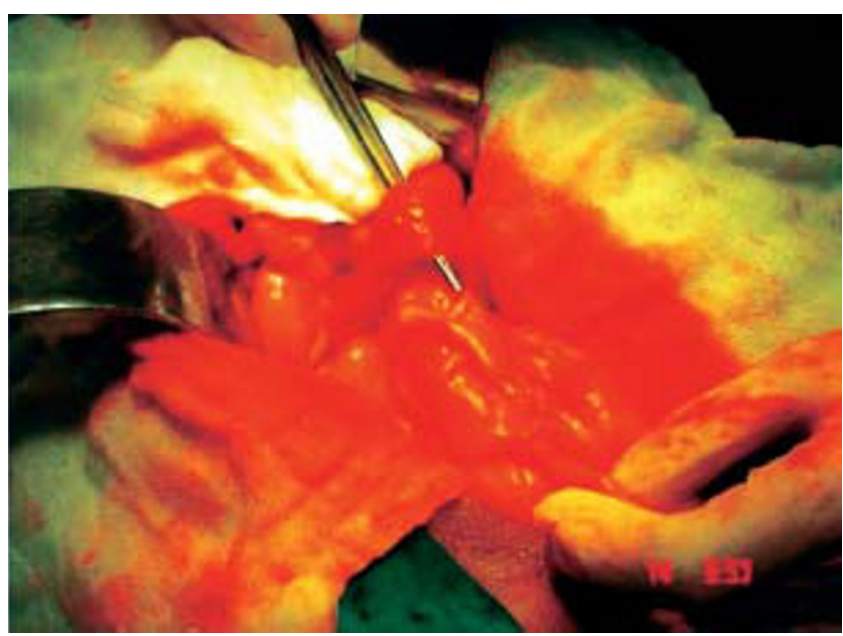

Fig. 2 : Caecum along with appendix withdrawn through a small rent in the jejunal mesentry just adjacent to the narrowed portion of jejunal segment

jejunum were dilated followed by a small narrowed segment of jejunum of about $8 \mathrm{~cm}$ in length. Close examination, revealed that most of the small intestine, caecum and part of ascending colon were lying in the upper half of peritoneal cavity in a collapsed state after passing through a small rent in the jejunal mesentery, adjacent to the narrowed portion of the jejunal segment (Fig. 2). There was no evidence of volvulus of the contents. The herniated portion of the intestine was withdrawn through the rent and repositioned in the peritoneal cavity. The narrowed segment of the jejunum was resected and end to end anastomosis performed. The resected portion of jejunum was sent for histopathological examination which revealed no abnormality. The caecum was fixed and laparotomy wound closed. The patient was discharged after ten days with complete recovery. On follow up, the patient was asymptomatic.

\section{Discussion}

Internal hernias are a rare event. An internal abdominal herniation is the protrusion of an abdominal organ through a normal or abnormal mesenteric or peritoneal aperture [2]. An internal hernia almost always remains within the peritoneal cavity. The most common form is the left para duodenal hernia through the para duodenal fossa of Lambert. Bowel may also herniate to the right of the duodenum through the mesentericoparietal fossa of Waldeyer through the foramen of Winslow into the lesser sac, congenital bands, congenital anomalies of the intestinal rotation and congenital defects in the mesentery or omentum [3]. Post surgical or traumatic defects of the mesentery and omentum predispose to internal herniation [4]. Literature reports of patients, in whom internal hernia of small bowel occurs after right lobe liver donor liver transplantation (LDLT). These patients have small bowel herniation with volvulus around the Roux-en-Y loop or hernia through the mesenteric window of transverse mesocolon after hepatico jejunostomy for the reconstruction [5].

In this case, probably the defect in the jejunal mesentery was congenital. The loops of bowel entered through the rent and remained there for a long time resulting in stretching and narrowing of the small bowel just adjacent to the mesenteric defect with out any histo pathological abnormality. Once the entire small bowel distal to the narrowed segment of jejunum passed through the defect, symptoms of obstruction set in. Usually the symptoms are non-specific and intermittent consisting of mild abdominal discomfort, alternating with episodes of intense colicky periumbilical pain, nausea and vomiting.

During asymptomatic intervals, no abnormality may be detected and routine investigations will be normal. $\mathrm{CT}$ scan after initial conventional radiography is preferred, before the patient is taken up for surgery [6]. CT criteria of internal hernia include clustering of small bowel loops, stretched and engorged mesenteric vessels with displacement of other bowel segments showing signs of intestinal obstruction [7]. Fixed small bowel loops adjacent to the abdominal wall, the lack of omental fat overlying the herniated bowel and central displacement of colon have been proposed as distinctive features of transmesenteric hernia [8]. Surgery is the treatment of choice. At laparotomy, the herniated contents are reduced, defect closed and depending upon the viability of the bowel appropriate surgical procedure is adopted.

\section{Conflicts of Interest}

None identified

\section{References}

1. Mathieu D, Luciani A. Internal Abdominal Herniation. Am J Radio 2004; 183: 397-404.

2. Miller PA, Mezwa DG, Feckzop PJ, Jafri ZH, et al. Imaging of abdominal hernias. Radiographics. 1995; 15: 333-47.

3. Black PR, Muller D, Crow J, Morris RC, Hussain AN. Mesenteric defects as a cause of intestinal volvulus with out normal rotation and as the possible primary etiology of intestinal atresia. J Pediatric Surg 1994; 29: 1339-43.

4. Ozenc A, Ozdemir A, Coskum T. Int Surg 1998; 83: 167-70.

5. Liv CL, Coc N, Chan SC, Fan ST, Wong J. Internal hernia of the small bowel after right lobe liver donor liver transplantation. Clinical Transplantation 2004; 18: 211-3.

6. Bert Lincoln, Pear MD, Alan Plunkelt L. Herniation of Ascending colon into the Lesser Sac. Radiology 2001; 218: 773-5.

7. Blacher A, Federle MP, Dodson SF. Internal hernia: Clinical and Imaging findings in 17 patients with emphasis of CT criteria. Radiology 2001; 218: 68-74.

8. Blacher A, Federle MP, Bran Catelli G, Peterson MS, Oliver $\mathrm{JH} 3^{\text {rd }}$, Li W. Radiologist performance in the diagnosis of internal hernia by using specific CT findings with emphasis on transmesenteric hernia. Radiology 2001; 221: 422-8. 